\title{
Mechanism of atomic force microscopy imaging of three-dimensional hydration structures at a solid-liquid interface
}

\author{
Takeshi Fukuma, ${ }^{1,2,{ }^{*}}$ Bernhard Reischl, ${ }^{3}$ Naritaka Kobayashi, ${ }^{1}$ Peter Spijker, ${ }^{4}$ Fillippo Federici Canova, ${ }^{5}$ \\ Keisuke Miyazawa, ${ }^{1}$ and Adam S. Foster ${ }^{1,4, \dagger}$ \\ ${ }^{1}$ Division of Electrical Engineering and Computer Science, Kanazawa University, Kanazawa 920-1192, Japan \\ ${ }^{2}$ ACT-C, Japan Science and Technology Agency, Saitama 332-0012, Japan \\ ${ }^{3}$ Nanochemistry Research Institute, Curtin Institute for Computation, Department of Chemistry, Curtin University, Perth, WA 6845, Australia \\ ${ }^{4}$ COMP Centre of Excellence, Department of Applied Physics, Aalto University, Helsinki FI-00076, Finland \\ ${ }^{5}$ Aalto Science Institute, Aalto University, Helsinki FI-00076, Finland
}

(Received 20 February 2015; revised manuscript received 4 August 2015; published 9 October 2015)

\begin{abstract}
Here we present both subnanometer imaging of three-dimensional (3D) hydration structures using atomic force microscopy (AFM) and molecular dynamics simulations of the calcite-water interface. In AFM, by scanning the 3D interfacial space in pure water and recording the force on the tip, a 3D force image can be produced, which can then be directly compared to the simulated 3D water density and forces on a model tip. Analyzing in depth the resemblance between experiment and simulation as a function of the tip-sample distance allowed us to clarify the contrast mechanism in the force images and the reason for their agreement with water density distributions. This work aims to form the theoretical basis for AFM imaging of hydration structures and enables its application to future studies on important interfacial processes at the molecular scale.
\end{abstract}

DOI: 10.1103/PhysRevB.92.155412

\section{INTRODUCTION}

Hydration plays fundamental roles in biomolecular functions [1-3], crystal growth [4], soil wetting [5], and catalytic reactions [6]. Despite the wide success of diffraction and spectroscopy techniques in investigating hydration structures at the nanoscale [7-13], molecular level detail of water distributions at critical inhomogeneous interfaces remains elusive. Recent developments in frequency modulation AFM (FM-AFM) [14] have enabled subnanometer-scale measurements of 3D force distributions at solid-liquid interfaces [15]. For instance, the experimentally measured force distributions at a mica-water interface show qualitative correlations with water density distributions (i.e., hydration structures) predicted by simulations $[15,16]$. These results suggest a possibility for direct imaging of hydration structures by FM-AFM. However, this argument has not generally been accepted due to the lack of clear explanation for the central issues of the imaging mechanism. In particular, it is a natural expectation that the scanning tip disturbs the hydration structures on the sample during imaging, convoluting the signal, and making direct visualization of intrinsic hydration structures almost impossible. The issue is further complicated by the influence of the hydration shell formed on the tip apex itself, and also that the overlap between local water density distributions on the tip and on the sample should induce rearrangements of the hydration structures on both sides.

To resolve this, it is imperative to investigate, in the same control system, the dynamic changes of the hydration structures of both the AFM tip and sample in experiment and

\footnotetext{
*fukuma@staff.kanazawa-u.ac.jp

†adam.foster@aalto.fi
}

Published by the American Physical Society under the terms of the Creative Commons Attribution 3.0 License. Further distribution of this work must maintain attribution to the author(s) and the published article's title, journal citation, and DOI.
PACS number(s): 68.37.Ps, 61.20.Ja, 68.35.Dv, 87.64.Dz

simulations by comparing the force $(F)$ and water density $(\rho)$ distributions, and identifying their correlation. As a substrate in this work we have chosen to use the (1014) surface of calcite $\left(\mathrm{CaCO}_{3}\right)$, which is an important material in biomineralization [17-19]. It has also been previously considered in high resolution AFM experiments [20-22] and simulations in liquids [23,24] due to its stability and candidacy as a benchmark system - even for some of the earliest AFM pioneers [25].

In simulations, to accurately model the complex interplay between tip, substrate, and solvent requires elaborate atomistic molecular dynamics (MD) simulations (in terms of size, duration, and the number of simulations required), far more complex as compared to similar simulations of AFM imaging in vacuum [26]. A more simple approach, where the tip itself is ignored [27,28], cannot be used in this systematic study as it excludes the inherently important interplay between hydration structures of both tip and sample, which, as we will show, are necessary to relate theoretical $F$ images and $\rho$ distributions [Fig. 1(b)] and experimental $F$ images [Fig. 1(d)]. The equilibrium hydration layer structure on calcite is highly dynamic, with complete exchange of water molecules in the first hydration layer on a nanosecond time scale. Obtaining the $F$ distributions requires free energy calculations due to the importance of the entropic contributions from the water molecules in the hydration layers of both tip and substrate $[23,29,30]$. Until now, only individual $F$ profiles over several ionic surfaces in water have been published [23,29-34], which is mainly due to the increased computational complexity involved when constructing a full 3D $F$ image from atomistic simulations. These individual $F$ profiles do not allow for good comparison with experiment, as the identity of the surface atoms over which the experimental profiles are obtained is unknown-only a 3D $F$ map allows for an unambiguous comparison. In this work, we computed free energy profiles as a function of tip-sample distance over $8 \times 4$ different surface sites in one calcite unit cell, allowing us to see lateral atomic-scale contrast in the hydration layer structures. The hydration structure of the surface itself is well known, with 


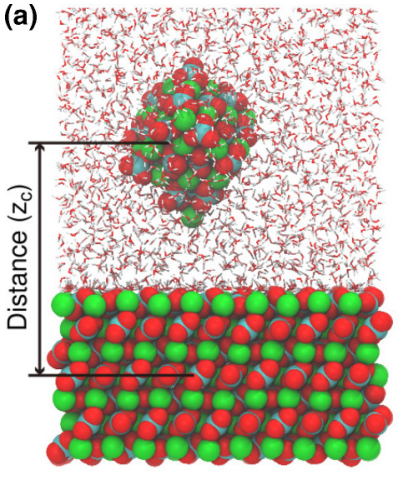

(c)
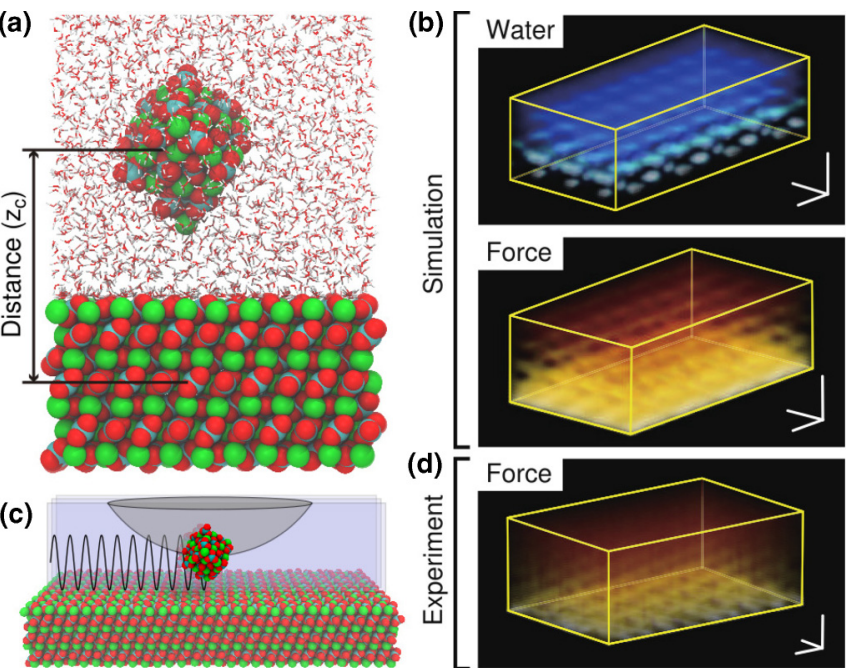

(d)

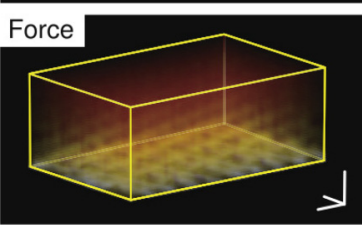

FIG. 1. (Color online) Three-dimensional $\rho$ and $F$ distributions obtained by simulation and experiment: (a) simulation model, where $z_{\mathrm{c}}$ is the $z$ component of the center-of-mass distance between the nanocluster tip and the sample slab; (b) 3D images of the simulated $\rho$ and $F$ distributions; (c) principle of 3D-SFM; and (d) 3D $F$ distribution experimentally measured by 3D-SFM. Scale bars, $0.5 \mathrm{~nm}$.

three distinct layers $[13,23]$ and the characteristic lateral ordering expected for ionic, amphoteric surfaces [35,36]. Hence, here we focus on understanding how this is interpreted in AFM.

FM-AFM measurements of 3D $F$ images in pure water, i.e., directly mimicking our MD simulation conditions, have to the best of our knowledge never been achieved before. All the 3D $F$ images reported so far were obtained in an electrolyte solution [15,16,37], and there is clear evidence of an improvement in contrast compared to pure water-in fact obtaining stable resolution at all in pure water is very challenging [20]. Here, we overcome this difficulty by using an ultrasmall cantilever in a 3D scanning force microscope (3D-SFM) [38]. Small cantilevers typically have a resonance frequency $\left(f_{0}\right)$ of $\sim 3.75 \mathrm{MHz}$ in water, which is approximately 25 times higher than a conventional cantilever $(\sim 150 \mathrm{kHz})$. This high $f_{0}$ enhances the averaging of the tip-water interactions, which inherently has considerable scattering, and provides sufficient stability and sensitivity for the $F$ measurements, enabling us to perform atomic-resolution $3 \mathrm{D} F$ measurements reproducibly [Fig. 1(d)].

\section{METHODS}

\section{A. Experimental methods}

The 3D $F$ image of the calcite-water interface was obtained by $3 \mathrm{D}-\mathrm{SFM}$ at room temperature. In 3D-SFM, the $z$ tip position is modulated with a sine wave while the tip is laterally scanned; see Fig. 1(c). During the scan, the average tip height is regulated to keep the average change in resonance frequency $\Delta f$ constant. By recording the $z$ control signal, we obtain a 2D height image. Meanwhile, the $\Delta f$ variation is also recorded with respect to the $3 \mathrm{D}$ tip position to produce a $3 \mathrm{D} \Delta f$ image. The 3D $\Delta f$ image was converted to the $3 \mathrm{D} F$ image using the Sader-Jarvis equation [39]. Height errors in the 3D $F$ image were corrected using the 2D height image and we removed the incomplete or distorted regions produced by the height correction and/or the tip drift. The size of the extracted 3D $F$ image was $3.67 \times 2.46 \times 1.43 \mathrm{~nm}^{3}$ with atomic-scale resolution.

A custom-built FM-AFM with an ultralow noise cantilever deflection sensor was used for the 3D-SFM imaging [40-42]. The 3D scan control and the data acquisition were performed by a commercially available AFM controller (ARC2, Asylum Research) with modifications in the software. We used an ultrashort cantilever (USC, Nanoworld) having a resonance frequency of $3.76 \mathrm{MHz}$, a spring constant of $69 \mathrm{~N} / \mathrm{m}$, and $\mathrm{Q}$ factor of 7.5 in water. A phase-locked loop circuit (OC4, SPECS) was used for detecting $\Delta f$ as well as for producing a cantilever excitation signal. The oscillation amplitude of the cantilever was kept constant at $0.11 \mathrm{~nm}$. The frequency and amplitude of the $z$ modulation during the 3D-SFM imaging were $200 \mathrm{~Hz}$ and $1.5 \mathrm{~nm}$, respectively. The lateral scan speed was $12.2 \mathrm{~nm} / \mathrm{s}$. Each $x z$ cross section was obtained in $0.32 \mathrm{~s}$ while the whole 3D image was obtained in $53 \mathrm{~s}$.

\section{B. Simulation methods}

The core of the simulation methodology has already been introduced previously [23]. Here, the atomistic model of the system consisted of a slab of calcite, 7 layers thick, exposing the (1014) surface along $z$ and a 160-atom calcite nanocluster, modeling the AFM tip apex, fully solvated by SPC/Fw water molecules in a rectangular simulation box with periodic boundaries, measuring $4.06 \times 3.95 \times 9.77 \mathrm{~nm}^{3}$. Atomistic interactions are described by the force field developed by Raiteri et al. [4]. We used the GROMACS simulation suite versions 4.5 and 4.6 [43] to carry out molecular dynamics simulations, and umbrella sampling to obtain free energy profiles. These profiles were calculated as a function of the tip-sample centers of mass distance $z_{\mathrm{c}}$ [see Fig. 1(a)], over $8 \times 4$ equally spaced different surface sites in one calcite unit cell. The range of the collective variable was initially sampled by 26 umbrella windows, with additional windows introduced where needed, to ensure good overlap. Every MD trajectory for each window was run for $6 \mathrm{~ns}$ in an NVT ensemble (after the height of the box had been equilibrated to obtain the correct bulk water density far from tip and surface), with a Nosé-Hoover thermostat maintaining the temperature at $300 \mathrm{~K}$. The first $250 \mathrm{ps}$ of the simulations were discarded in the analysis to decorrelate from the starting configuration. The 160 -atom nanocluster used as a tip model showed increased stability over the 125-atom nanocluster used previously [23], allowing us to sample the tip apex-sample separation distance from over $1 \mathrm{~nm}$ all the way to hard contact. We repeated the free energy calculations for two orientations of the cluster, terminating in either a $\mathrm{Ca}^{2+}$ or $\mathrm{CO}_{3}^{2-}$ ion. As the time scale of water relaxation is several orders of magnitude faster than the tip oscillation, the derivative of the free energy profiles with respect to the tip-sample distance gives an $F$ profile that should be a very good approximation to similar $F$ profiles from AFM experiments-where the measured signal is a time average over many oscillation cycles at the same lateral position. Hence, the $3 \mathrm{D}$ map of the effective force on the AFM tip was obtained from the free energy derivative with respect to $z_{\mathrm{c}}$. A running average along $z$ was performed on 
(a)

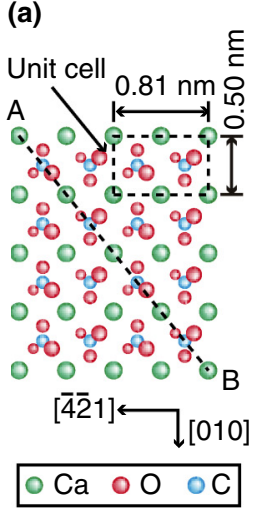

(b) $A$

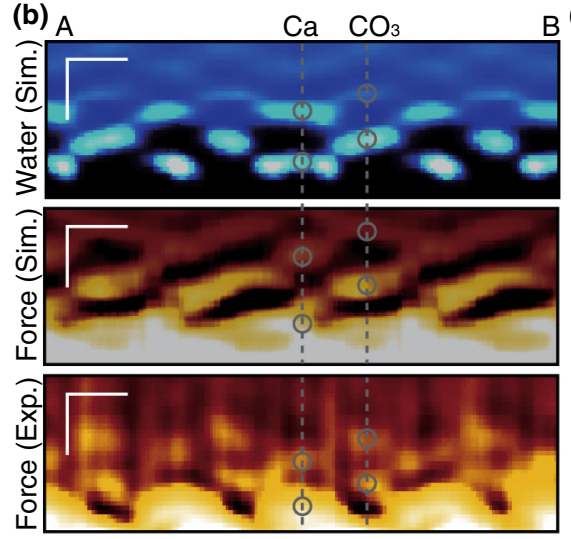

(e)

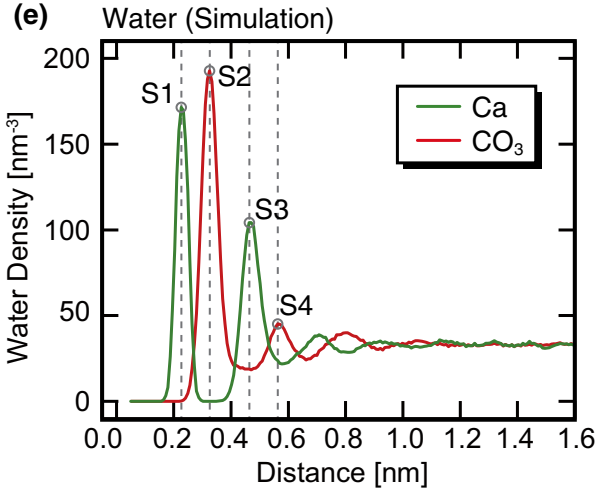

(c) Water (Sim.) Force (Sim.)

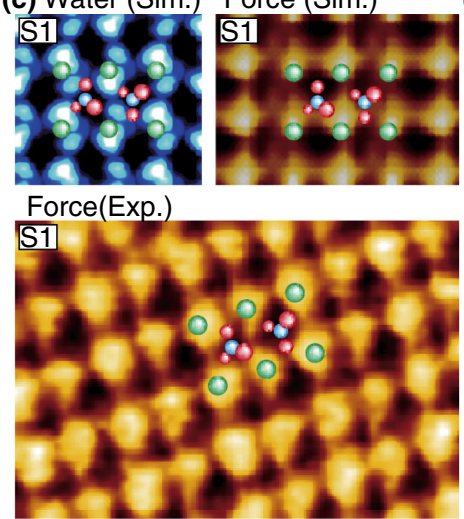

(f) Force (Simulation)

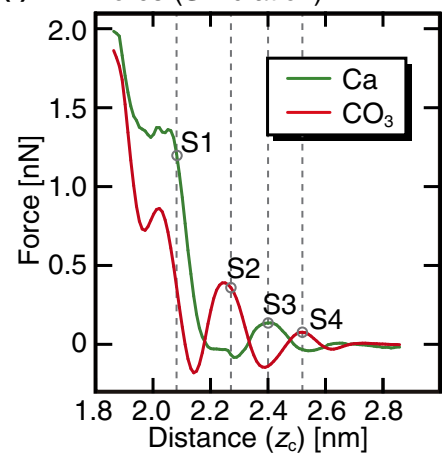

(d) Water (Sim.) Force (Sim.)

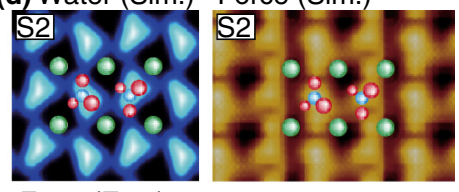

Force(Exp.)

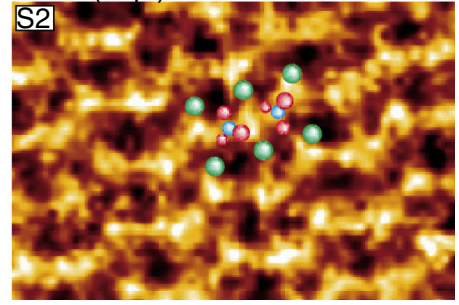

(g) Force (Experiment)

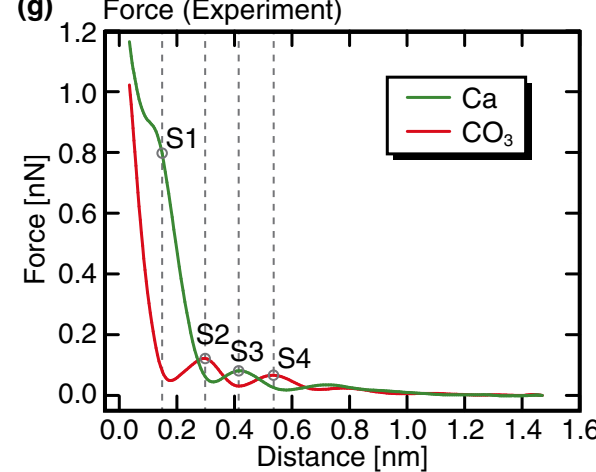

FIG. 2. (Color online) Comparison between the simulated 3D $\rho$ and $F$ images and the experimentally measured 3D $F$ image: (a) model of $\mathrm{CaCO}_{3}(10 \overline{1} 4)$ surface; (b) $z$ cross sections, taken along the line A-B from (a). Scale bars, $0.3 \mathrm{~nm}$. (c) and (d) $x y$ cross sections, and (e) $-(\mathrm{g}) z$ profiles measured at $\mathrm{Ca}$ and $\mathrm{CO}_{3}$ sites.

each $F$ profile to reduce noise, and a cubic spline was then used to interpolate laterally between neighboring data points. For the atomistic analysis, snapshots from atomic positions in the original umbrella sampling trajectories where rebinned in $z_{\mathrm{c}}$ intervals of $0.02 \mathrm{~nm}$. In order to be able to compare the 3D $F$ map to the equilibrium 3D water $\rho$ distribution, we also ran one simulation similar to the ones described above, but without the tip present.

\section{RESULTS}

\section{A. Comparison between simulation and experiments}

Figure 2 shows a direct comparison between the experimentally measured 3D $F$ images and the simulated 3D $\rho$ and $F$ distributions. Owing to the full 3D $F$ information from both experiment and simulation, we are able to determine the positions of the $\mathrm{Ca}^{2+}$ and $\mathrm{CO}_{3}^{2-}$ ions in the surface by optimizing the agreement between the $x y$ and $z$ cross sections; see Figs. 2(b)-2(d). After alignment, the $\rho$ profiles at the $\mathrm{Ca}$ and $\mathrm{CO}_{3}$ sites alternately show peaks [see Fig. 2(e)], and we refer to the four different hydration layers corresponding to these peaks as $\mathrm{S} 1-\mathrm{S} 4$. Similarly, in the $F$ profiles over the same sites, these peaks can also be found in both simulation and experiment; see Figs. 2(f) and 2(g). As there are two nonequivalent $\mathrm{Ca}$ and $\mathrm{CO}_{3}$ sites [see Fig. 2(a)], the simulated profiles are averaged over the two sites, while the experimentally measured profiles are averaged over all the $\mathrm{Ca}$ and $\mathrm{CO}_{3}$ sites. Unlike the $\rho$ profile, the $F$ profiles show a sharp increase near the calcite surface which is due to the direct interaction of the substrate with the tip. Although the $F$ values obtained by simulation are slightly lower than those obtained by experiment, they are of the same order of magnitude, which gives us confidence that the interactions within the theoretical model are physically representative.

Although small differences exist between the different $z$ cross sections for the $\rho$ and $F$ distributions over $\mathrm{Ca}$ and $\mathrm{CO}_{3}$ sites [see Fig. 2(b)], the alternate peaks are still commonly found over $\mathrm{Ca}$ and $\mathrm{CO}_{3}$ sites (circles). Also, the $F$ images show an enhanced contrast near the surface due to the aforementioned substrate influence. In the simulated $x y$ cross sections [Fig. 2(c)] obtained at the $z$ position corresponding to $\mathrm{S} 1$, the Ca sites show a higher contrast than the $\mathrm{CO}_{3}$ sites. In the simulated $x y$ cross sections at S2 [Fig. 2(d)] an inverted contrast can be seen, confirming the existence of alternate peaks on the $\mathrm{Ca}$ and $\mathrm{CO}_{3}$ sites. These major contrast features (i.e., alternating peaks) in the simulated $\rho$ and $F$ images are confirmed in the experimentally measured $F$ images [larger panels of Figs. 2(c) and 2(d)]. This striking agreement between simulation and experiments on one hand reinforces the validity of the simulations, but on the other hand also shows the strong correlation between the $F$ profiles and the intrinsic hydration layer structure on calcite, in the absence of a tip.

\section{B. Atomic-scale imaging mechanism}

The major contrast features (i.e., the alternating peaks) in the experimentally measured $F$ image are reproduced well in the simulation. Thus, at least for the alternating peaks, we 
(a)

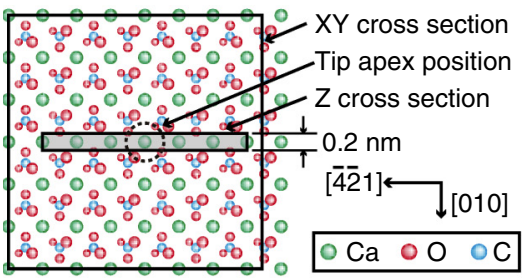

(b)
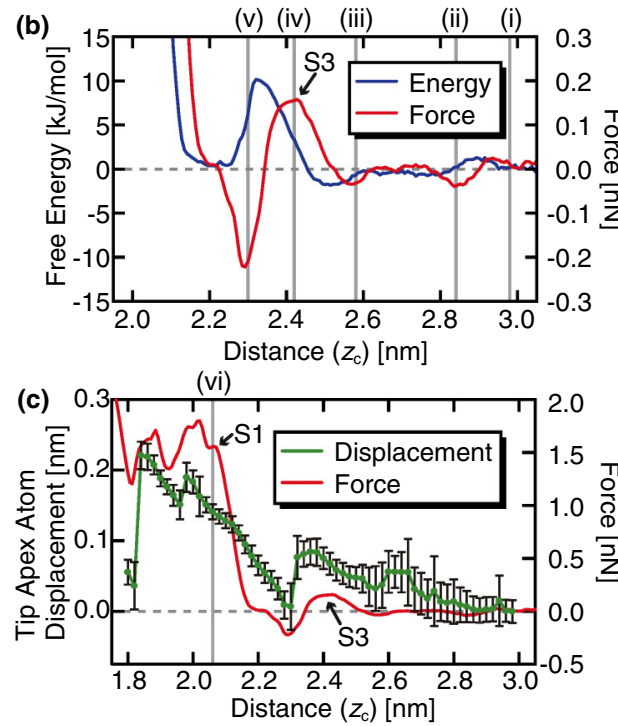

(d)

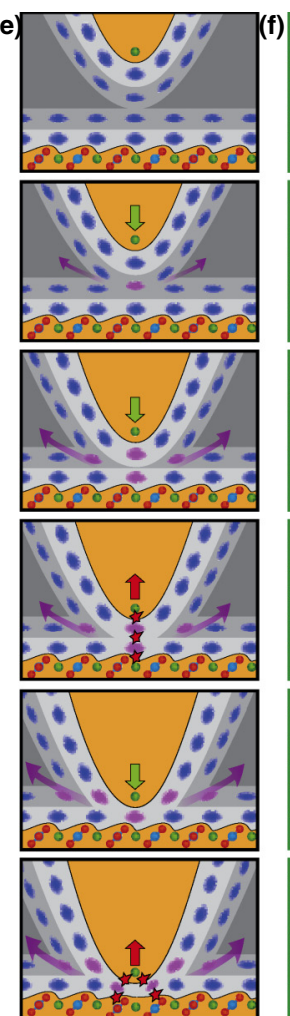

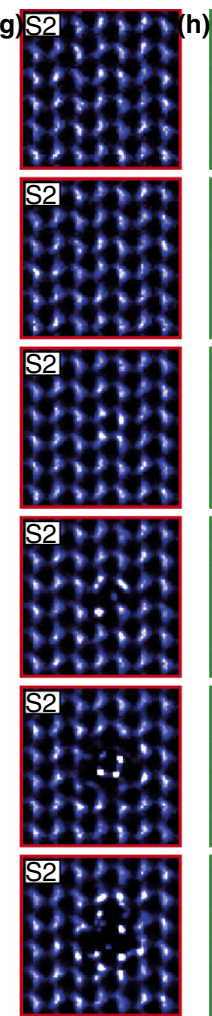


FIG. 3. (Color online) Analysis of the $z_{\mathrm{c}}$-dependent change of the simulated 3D $\rho$ distribution during the tip approach over a Ca site: (a) model of a calcite (1014) surface indicating the relevant $x y$ and $z$ cross sections [also see Fig. 2(e)]; (b) $z$ profiles of the free energy and force $F$; (c) $z$ profiles of the tip deformation $\Delta z_{a}$ and force $F$ (error bars $\pm\left\langle\Delta z_{a}^{2}\right\rangle$ ); (d) $z$ cross sections of the simulated 3D $\rho$ distribution; (e) schematic diagram of the dominant processes in the contrast mechanism. The spots indicate water localized by the tip and surface (blue), with the water ordering indicated by grayscale, from highly ordered (light gray) to bulklike (dark gray). In general, as the tip approaches, ordered water is forced into the bulk region (pink arrows), producing an attractive entropic contribution. When tip and surface hydration layers match up (purple spots), the repulsive potential contribution is significantly reduced and the tip apex displaces towards the surface (green arrow). When hydration layers are forced against each other and the tip/surface (red stars), there is a strong repulsive potential contribution to the free energy and the tip apex displaces away from the surface (red arrow); (f)-(h) $x y$ cross sections of the simulated 3D $\rho$ distribution.

should be able to discuss their formation mechanism based on the simulation results. For this purpose, here we investigate changes in the hydration structure around the tip apex during the tip approach over a $\mathrm{Ca}$ site and their correlation with the induced free energy and $F$ variations (Fig. 3).

Figure 3(a) shows the crystal structure of a cleaved calcite surface, where a circle with a dotted line shows the lateral position of the tip apex $\mathrm{Ca}$ ion, while Fig. 3(b) shows the associated $F$ and free energy versus $z_{\mathrm{c}}$ curves. We obtained numerous $x y$ and $z$ cross sections of the simulated 3D $\rho$ image at every $20 \mathrm{pm}$ in $z_{\mathrm{c}}$ (see videos in Supplemental Material [44]), but in Figs. 3(d) and 3(f)-3(h), we show only six of them to highlight important events taking place during the tip approach. The lateral position of the cross sections is indicated by the rectangle in Fig. 3(a). Figure 3(e) shows simplified models of the $z$ cross sections shown in Fig. 3(d), and effectively summarizes the entire contrast mechanism we discuss in the following.

\section{Distance dependence}

At position (i) [see Fig. 3(b)], $z_{\mathrm{c}}=2.98 \mathrm{~nm}$, the tip apex is $\sim 1 \mathrm{~nm}$ away from the surface and the hydration structures on the tip and the sample are still in their intrinsic equilibrium states. This can be seen in the $z$ cross section [Fig. 3(d)(i)], where we can identify the $\rho$ distributions $\mathrm{S} 1$ and $\mathrm{S} 3$ on the $\mathrm{Ca}$ sites and the first and the second hydration shells on the tip (T1 and T2, respectively). In the same figure, the green circle overlaid on the tip apex atom (T0) indicates the ionic radius of $\mathrm{Ca}^{2+}$, while the red one is the sum of the ionic radii of $\mathrm{Ca}^{2+}$ and the water oxygen, representing a reasonable estimate of the steric repulsion range between $\mathrm{T} 0$ and the surrounding water molecules.

As the tip approaches, T2 starts to overlap with S3 at position (ii). This leads to a reduction of the free energy and produces a small attractive $F$ peak. Despite the overlap between $\mathrm{T} 2$ and $\mathrm{S} 3$, the lateral distribution of $\mathrm{S} 3$ shows almost no disturbance.

At position (iii), T1 starts to overlap with S3, leading to a reduction of the free energy and producing an attractive $F$ peak. The enhanced $\rho$ distribution corresponding to T1 can be clearly identified within the $x y$ cross section of $\mathrm{S} 3$, whereas $\mathrm{S} 2$ and $\mathrm{S} 1$ remain unaffected by the presence of the tip.

While approaching from 2.5 to $2.3 \mathrm{~nm}$, the free energy shows a sharp increase, with the $F$ profile showing a repulsive peak at position (iv). During this approach, the tip apex atom T0 is pushed upward, as the water molecules under T0 are increasingly confined and start to rearrange in the combined 
T1/T2/S3 hydration structures (see discussion in Sec. III B 3). Only minor distortions appear in S2.

As the tip approaches beyond the free energy peak at $2.32 \mathrm{~nm}$, T1 starts to overlap with S1, effectively reforming the intrinsic hydration structure of the tip apex. This event leads to a sharp decrease of the free energy and produces the large attractive $F$ peak at position (v). At this distance, water molecules in $\mathrm{S} 1$ start to be pinned between the tip and surface.

After the removal of the enhanced $\rho$ distribution under T0, the free energy rapidly decreases to nearly zero and remains small from 2.26 to $2.14 \mathrm{~nm}$. In this $z_{\mathrm{c}}$ range, the $\rho$ distributions in $\mathrm{S} 2-\mathrm{S} 4$ are being heavily disturbed, and a considerable volume of water is removed from the interface and released to the bulk due to further tip penetration into the interfacial space. Nevertheless, the free energy profile shows little influence of these events in this distance range, suggesting that the energetic and entropic contributions from the different processes cancel out (see discussion in Sec. III B 2).

Upon further tip approach, the free energy starts to increase steeply when T0 penetrates $\mathrm{S} 1$. This leads to a sharp increase of $F$ from 2.16 to $2.08 \mathrm{~nm}$. Even in this $z_{\mathrm{c}}$ range, as the water molecules are increasingly pinned under the tip, most of S1 remains intact, indicating that the hydration peaks in $\mathrm{S} 1$ are strongly bound to the sample Ca sites.

Below position (vi), the free energy increases at a nearly constant rate and hence the $F$ profile reaches a constant, but large value. This $z_{\mathrm{c}}$ range corresponds to the tip coming into hard contact with the surface, and the displacement of the tip and sample atoms, as well as the reorganization of the lateral distribution in $\mathrm{S} 1$ prevent a further increase of $F$.

\section{Overlap between tip and sample hydration peaks}

Hydration layers at a solid-liquid interface form due to the interactions between water molecules and surface atoms [see Fig. 3(e), where the locations to find water molecules with the highest probability are colored blue]. A site with an enhanced $\rho$ distribution (i.e., hydration peak) corresponds to an energetically favorable spot for a water molecule. In a previous study, Harada et al. [31] investigated the correlation between overlap of hydration layers and the induced free energy change for two opposing flat surfaces under various conditions. They found that the overlap between the two hydration layers always results in a reduction of the free energy and called this effect "constructive interference." This previous work suggests that the energetic merit obtained by the interaction with two surfaces generally exceeds the disadvantage caused by the removal of interfacial water.

Other theoretical investigations by Watkins et al. [27,29] on the entropic and potential contributions to the free energy change during a tip approach to a sample surface, showed an increasing repulsive potential energy contribution as the tip pushes through the water layers. However, this is largely compensated by an increase of entropy as originally structured water is pushed into the unstructured bulk region.

These effects can be seen in detail in our results and combine to underpin the change in free energy as the tip approaches. When two opposing surfaces approach, density peaks within the intrinsic hydration layers of the respective surfaces overlap and energetically favorable spots are shared between the two surfaces [Fig. 3(e), positions (ii, iii, v)]. Thus, a water molecule in the overlapping region can simultaneously interact with both surfaces, lowering the energy compared to the interaction with a single surface. Simultaneously, the overlap also implies the removal of another interfacial water molecule ("the extra") into bulk, which is energetically unfavorable, but leads to a gain in entropy. Our simulation results show that overlap and sharing between the tip and sample hydration structures leads to a reduction of the free energy and produces an attractive peak in the $F$ profile.

\section{Confinement and displacement of hydration peaks}

There has been some debate on whether a strongly bound water molecule at the apex of a hydrophilic AFM tip can act as the actual tip termination during hydration force measurements $[27,28]$. If this were the case, $T 1$ [the hydration peak at the tip apex (see Fig. 3(d)], and not T0 (the tip apex) would interact directly with a sample hydration peak when we detect a corresponding repulsive $F$ peak. Our simulation results show that repulsive $F$ peaks appear when the tip apex atom T0 interacts directly with S3 or S1. The changes in free energy observed when $\mathrm{T} 1$ interacts with $\mathrm{S} 3$ are much smaller, and induce repulsive $F$ values that may not even be detectable in experiment.

When T0 interacts with S1 or S3, the water distribution under $\mathrm{T} 0$ is confined between $\mathrm{T} 0$ and the sample surface and pushed down for a relatively long distance. This behavior is illustrated in Fig. 3(e) (iv, vi) [see also videos in Supplemental Material (T0-S3.mp4 and T0-S1.mp4)]. Such an interaction both increases the potential energy and reduces the entropy of the water molecules confined between the tip and the sample, leading to an increase of the total free energy and, hence, producing a repulsive $F$ peak. In contrast, when T0 interacts with S2 at position (v), there is no hydration peak located directly under $\mathrm{T} 0$, and, consequently, the confinement of water is weaker. The water distributions adjacent to $\mathrm{T} 0$ are laterally displaced without showing any significant persistence during the tip approach [see the video in Supplemental Material (T1-S1.mp4)].

Therefore, the repulsive $F$ peaks originate from the increasing confinement of the water distribution under the approaching tip, until a reorganization of the hydration structure occurs. Once this change is induced, the free energy decreases, as water molecules find energetically favorable positions in the new combined hydration structure, or get expelled to the bulk, lowering the free energy through entropy gain.

\section{Predominance of the force on the tip apex}

The vertical displacement of the tip apex atom T0 with respect to the tip center of mass $\left(\Delta z_{\mathrm{a}}\right)$ is plotted in Fig. 3(c) along with the force $F$ acting on the tip, obtained from the derivative of the free energy. Assuming that the apex atom can be seen as being attached to a rigid tip body with a harmonic spring, $\Delta z_{\mathrm{a}}$ should be roughly proportional to $F_{\mathrm{a}}$, the force acting on T0. Additionally, $\delta z_{\mathrm{a}}$, the standard deviation of the vertical tip displacement (error bars on the $\Delta z_{\text {a }}$ profile), is a good measure for the vertical confinement of the tip apex. From Fig. 3(c) it can be seen that the $F$ and $\Delta z_{\text {a }}$ profiles are indeed in very good agreement: As $F$ increases, $\Delta z_{\mathrm{a}}$ increases and $\delta z_{\mathrm{a}}$ decreases. Once over the repulsive force peak, $\Delta z_{\mathrm{a}}$ decreases 
and $\delta z_{\mathrm{a}}$ increases again. This once again confirms that the repulsive $F$ peaks originate in the confinement of water under the tip, pushing the tip apex atom upward. Once the barrier is crossed, the tip apex can relax to its equilibrium structure. The strong correlation between $\Delta z_{\mathrm{a}}$ and $F$ indicates that the force on the tip strongly depends on the force on the apex atom, which explains the high sensitivity of the AFM tip to changes in hydration structures directly underneath the tip apex.

\section{DISCUSSION}

\section{A. Atomic resolution}

The atomic-resolution imaging mechanism of scanning tunneling microscopy (STM) [45] is explained by the strong distance dependence of the tunneling current flowing between a tip and a sample. If we have an atomic-scale protrusion at the tip apex, the exponential decay length of the current means that it is dominated by tunneling between the atom at the tip apex and the closest atom in the surface-hence we can obtain an image with atomic resolution.

As discussed previously, the atomic-scale imaging mechanism of a hydration structure by AFM is explained by the free energy change induced by the hydration peak overlap, confinement, and removal events. The free energy does not behave monotonously (nor have local decay in the same sense as the tunneling current), since the free energy events are determined by the nature of the specific tip and surface hydration structures. However, the free energy contribution of events just under T0 dominate, and, for an atomically sharp tip, result in atomically resolved structure in AFM.

\section{B. Tip termination}

The discussions above are based on the simulation results obtained with a Ca-terminated tip above a $\mathrm{Ca}$ site. To investigate the generality of the proposed imaging mechanism, we also performed similar simulations of a 3D $F$ image with the same Ca-terminated tip over a $\mathrm{CO}_{3}$ site (Fig. S2 in Supplemental Material), and with a $\mathrm{CO}_{3}$-terminated tip above both $\mathrm{Ca}$ and $\mathrm{CO}_{3}$ sites (Figs. S3 and S4 in Supplemental Material).

In all the cases, our main claims on the atomic-scale imaging mechanism of a 3D hydration structure are consistently supported by the simulation results, although minor differences exist between the images obtained by the $\mathrm{Ca}$ and $\mathrm{CO}_{3}$ tips.

The main difference that we found between the two tip models was that the $\mathrm{CO}_{3}$ ion at the apex showed more lateral freedom of movement, in addition to being able to rotate slightly, compared to the Ca-terminated tip. Thus, when hydration peaks are confined between the apex $\mathrm{CO}_{3}$ ion and the sample surface, the ion can easily rotate or shift in $x y$ directions, releasing the pressure under the tip. Consequently, the attractive and repulsive force peaks measured with the $\mathrm{CO}_{3}$ tip are much smaller than that with the Ca tip, over both the $\mathrm{Ca}$ and $\mathrm{CO}_{3}$ sites. For example, the repulsive peak forces corresponding to $\mathrm{S} 1$ and $\mathrm{S} 3$ over a $\mathrm{Ca}$ site are 1.5 and $0.2 \mathrm{nN}$ for the $\mathrm{Ca}$ tip while 0.3 and $0.1 \mathrm{nN}$ for the $\mathrm{CO}_{3}$ tip, respectively. Thus, a more flexible tip apex can overcome the barrier presented by the hydration peak with a relatively small force.

\section{Tip sharpness}

In the analysis presented so far, we used relatively sharp tips. For a blunter tip, we expect that the volume of the water removed from the interface and the direct long-range interaction between the tip and the sample should increase. However, these contributions should not have significant influence on the local atomic-scale $F$ contrasts, but only on the long-range background $F$ profile. Extremely blunt tips may have a few tip apex atoms at the same height, causing the confinement of hydration peaks to take place under several apex atoms at once, complicating the relationship between the measured $F$ distribution and the intrinsic hydration structure. However, such tips can be identified and excluded beforehand, as they would not yield a clear atomic-scale contrast in an experimental 2D AFM image of a sample surface either.

\section{Tip material}

We have used an approximately cubic calcite nanocluster, rotated on a corner, to model the AFM tip. Actual tips are usually made of silicon, which is covered in an amorphous oxide layer, and will form silanol groups in water. The argument for choosing the same material for the tip and surface, in addition to reducing the number of interaction parameters in the simulation, is that the tip either could have picked up material from the surface during an initial hard contact, or that ions that have dissolved from the surface have covered the tip apex. While the choice of tip material certainly affects the simulation results, we are confident that a simulation with another hydrophilic cluster, such as silanol-terminated silicon dioxide, would show very similar results (hydration layer formation on a silica tip is similar [46]). Our choice implies that the interaction of water molecules with both the tip and surface is equally strong. Using a tip material with less ordered hydration layers compared to calcite, would lead to smaller variations in the free energy profiles, and smaller force amplitudes, but the imaging mechanism would remain similar.

\section{CONCLUSIONS}

In this study, we obtained 3D $F$ images at a calcite-water interface by MD simulation and 3D-SFM experiments. For the simulation, we presented a full 3D $F$ image obtained by an atomistic MD simulation with an explicit tip model for the first time. For the experiments, we reported an atomic-scale 3D $F$ image obtained in pure water for the first time. Combining these efforts, we enabled direct comparison between the simulated and experimentally measured 3D $F$ images. The results indicate that both the images consistently show the alternating peaks corresponding to the sample hydration peaks (S1-S4). This agreement confirms the strong similarity between the $F$ and $\rho$ images, and ensures the validity of the simulation results.

We investigated changes in the hydration structure around the tip apex during a tip approach process, and their correlation with the induced free energy and $F$ variations. From these results, we clarified the atomic-resolution imaging mechanism of a hydration structure by AFM. The overlap, confinement and removal of hydration peaks between the tip apex atom and the sample surface predominantly contributes to the short-range 
free energy and $F$ variations. Thus, we can obtain atomic-scale $F$ contrasts reflecting the intrinsic hydration structure despite the disturbances induced by a nanoscale tip.

So far, 3D hydration structure measurements by AFM have not been widely accepted due to the lack of clear explanation of the central issues of the imaging mechanism. By combining the efforts in simulation and experiments, we have presented detailed explanations of these issues and clarified the imaging mechanism. This work should form the theoretical foundation for this technique and stimulate its future applications in various scientific fields.

\section{ACKNOWLEDGMENTS}

This work was supported by KAKENHI (Grant No. 25706023), Japan Society for the Promotion of Science; ACT-C, Japan Science and Technology Agency; the Academy of Finland through its Centres of Excellence Program (Project No. 251748) and FP7 project PAMS. B.R. has been supported by the Finnish Cultural Foundation (SKR), EMRP project MechProNO, and the Australian Research Council (DP140101776). We acknowledge use of the supercomputer resources of the Finnish CSC-IT Center for Science.
[1] J. Ostmeyer, S. Chakrapani, A. C. Pan, E. Perozo, and B. Roux, Nature (London) 501, 121 (2013).

[2] D. Krepkiy, M. Mihailescu, J. A. Freites, E. V. Schow, D. L. Worcester, K. Gawrisch, D. J. Tobias, S. H. White, and K. J. Swartz, Nature (London) 462, 473 (2009).

[3] M. S. Cheung, A. E. García, and J. N. Onuchic, Proc. Natl. Acad. Sci. 99, 685 (2002).

[4] P. Raiteri and J. D. Gale, J. Am. Chem. Soc. 132, 17623 (2010).

[5] P.-Y. Meslin, O. Gasnault, O. Forni, S. Schröder, A. Cousin, G. Berger, S. M. Clegg, J. Lasue, S. Maurice, V. Sautter et al., Science 341, 1238670 (2013).

[6] G. Dong, P. Teo, Z. K. Wickens, and R. H. Grubbs, Science 333, 1609 (2011).

[7] K. B. Eisenthal, Chem. Rev. 96, 1343 (1996).

[8] L. Cheng, P. Fenter, K. L. Nagy, M. L. Schlegel, and N. C. Sturchio, Phys. Rev. Lett. 87, 156103 (2001).

[9] B. Bagchi, Chem. Rev. 105, 3197 (2005).

[10] S. K. Pal and A. H. Zewail, Chem. Rev. 104, 2099 (2004).

[11] M. E. Johnson, C. Malardier-Jugroot, R. K. Murarka, and T. Head-Gordon, J. Phys. Chem. B 113, 4082 (2009).

[12] B. Jana, S. Pal, and B. Bagchi, J. Chem. Sci. 124, 317 (2012).

[13] P. Fenter, S. Kerisit, P. Raiteri, and J. D. Gale, J. Phys. Chem. C 117, 5028 (2013).

[14] F. Giessibl, Rev. Mod. Phys. 75, 949 (2003).

[15] T. Fukuma, Y. Ueda, S. Yoshioka, and H. Asakawa, Phys. Rev. Lett. 104, 016101 (2010).

[16] K. Kobayashi, N. Oyabu, K. Kimura, S. Ido, K. Suzuki, T. Imai, K. Tagami, M. Tsukada, and H. Yamada, J. Chem. Phys. 138, 184704 (2013).

[17] M. D. Hollingsworth, Science 326, 1194 (2009).

[18] Y.-Y. Kim, K. Ganesan, P. Yang, A. N. Kulak, S. Borukhin, S. Pechook, L. Ribeiro, R. Kröger, S. J. Eichhorn, S. P. Armes et al., Nat. Mater. 10, 890 (2011).

[19] F. Natalio, T. P. Corrales, M. Panthofer, D. Schollmeyer, I. Lieberwirth, W. E. G. Muller, M. Kappl, H. J. Butt, and W. Tremel, Science 339, 1298 (2013).

[20] S. Rode, N. Oyabu, K. Kobayashi, H. Yamada, and A. Kuehnle, Langmuir 25, 2850 (2009).

[21] H. Imada, K. Kimura, and H. Onishi, Langmuir 29, 10744 (2013).

[22] C. Marutschke, D. Walters, J. Cleveland, I. Hermes, R. Bechstein, and A. Kühnle, Nanotechnology 25, 335703 (2014).
[23] B. Reischl, M. Watkins, and A. S. Foster, J. Chem. Theory Comput. 9, 600 (2013).

[24] M. Ricci, P. Spijker, F. Stellacci, J.-F. Molinari, and K. Voïtchovsky, Langmuir 29, 2207 (2013).

[25] F. Ohnesorge and G. Binnig, Science 260, 1451 (1993).

[26] C. Barth, A. S. Foster, C. R. Henry, and A. L. Shluger, Adv. Mater. 23, 477 (2011).

[27] M. Watkins and B. Reischl, J. Chem. Phys. 138, 154703 (2013).

[28] K.-I. Amano, K. Suzuki, T. Fukuma, O. Takahashi, and H. Onishi, J. Chem. Phys. 139, 224710 (2013).

[29] M. Watkins and A. L. Shluger, Phys. Rev. Lett. 105, 196101 (2010).

[30] M. Watkins, M. L. Berkowitz, and A. L. Shluger, Phys. Chem. Chem. Phys. 13, 12584 (2011).

[31] M. Harada and M. Tsukada, Phys. Rev. B 82, 035414 (2010).

[32] M. Tsukada, N. Watanabe, M. Harada, and K. Tagami, J. Vac. Sci. Technol. B 28, C4C1 (2010).

[33] D. Argyris, P. D. Ashby, and A. Striolo, ACS Nano 5, 2215 (2011).

[34] D. Argyris, A. Phan, A. Striolo, and P. D. Ashby, J. Phys. Chem. C 117, 10433 (2013).

[35] J. C. Fogarty, H. M. Aktulga, A. Y. Grama, A. C. T. van Duin, and S. A. Pandit, J. Chem. Phys. 132, 174704 (2010).

[36] M. Sulpizi, M.-P. Gaigeot, and M. Sprik, J. Chem. Theory Comput. 8, 1037 (2012).

[37] H. Asakawa, S. Yoshioka, K.-I. Nishimura, and T. Fukuma, ACS Nano 6, 9013 (2012).

[38] T. Fukuma, K. Onishi, N. Kobayashi, A. Matsuki, and H. Asakawa, Nanotechnology 23, 135706 (2012).

[39] J. E. Sader and S. P. Jarvis, Appl. Phys. Lett. 84, 1801 (2004).

[40] T. Fukuma, M. Kimura, K. Kobayashi, K. Matsushige, and H. Yamada, Rev. Sci. Inst. 76, 053704 (2005).

[41] T. Fukuma and S. P. Jarvis, Rev. Sci. Inst. 77, 043701 (2006).

[42] T. Fukuma, Rev. Sci. Inst. 80, 023707 (2009).

[43] D. Van Der Spoel, E. Lindahl, B. Hess, G. Groenhof, A. E. Mark, and H. J. C. Berendsen, J. Comput. Chem. 26, 1701 (2005).

[44] See Supplemental Material at http://link.aps.org/supplemental/ 10.1103/PhysRevB.92.155412 for video files showing continuous water distribution changes during the tip approach to the sample surface.

[45] W. Hofer, A. Foster, and A. Shluger, Rev. Mod. Phys. 75, 1287 (2003).

[46] S. Morita, F. J. Giessibl, E. Meyer, and R. Wiesendanger (eds.), Noncontact Atomic Force Microscopy, NanoScience and Technology (Springer International Publishing, Cham, 2015). 